\title{
Genoproteomic analysis of leukemic cell response to Cytarabine reveals synergistic opportunities centered on cholesterol metabolism
}

\begin{abstract}
Alex Farmer, Fangli Chen, Xue Wu, Adriana Rogozea, Mircea Ivan and Heiko Konig Melvin and Bren Simon Cancer Center, Indiana University School of Medicine, Indianapolis, IN

Background: Patients with acute myeloid leukemia (AML) carry a dismal prognosis due to drug resistant cancer cells that reside in $\mathrm{O}_{2}$ deprived niches of the bone marrow. Here, we sought to interrogate $A M L$ drug responses under hypoxic $\left(1 \% \mathrm{O}_{2}\right)$ and normoxic $\left(21 \% \mathrm{O}_{2}\right)$ conditions in order to identify novel drug targets.
\end{abstract}

Methods: We utilized RNAseq, PCR and RPPA to interrogate chemotherapy-induced expression responses in M14 cells. We further assessed intracellular cholesterol levels (ICCLs), cell growth and apoptotic cell death per MTT and FACS analysis in AML cell lines and primary cells (PCs) in response to chemotherapy under normoxia and hypoxia.

Results: Cytarabine blunted cholesterol biosynthesis- and induced CD36-mRNA expression under $1 \%$ and $21 \% \mathrm{O}_{2}$. ICCLs were significantly higher in Cytarabine treated AML cells compared to untreated controls. Treatment with Rosuvastatin significantly inhibited growth of M14, THP1, OCI-AML3 as well as AML PCs $(n=3)$. Further, Rosuvastatin lowered ICCLs and conferred pro-apopotic and growth inhibitory effects against M14 and THP-1 cells. Growth inhibition was enhanced when Rosuvastatin was combined with Cytarabine, yielding additive to synergistic effects. Similar effects were observed in AML PCs $(n=10)$ where Rosuvastatin combined with Cytarabine demonstrated strong synergy.

Conclusion: (i) Depletion of ICCLs is counterbalanced by CD36 expression in Cytarabinetreated AML cells under normoxia and hypoxia. (ii) Rosuvastatin exerts antileukemic activity in AML cell lines and PCs via downregulation of ICCLs, induction of apoptosis and inhibition of cell growth. (iii) Rosuvastatin acts synergistically with Cytarabine against AML cells. Impact: Further investigation of Rosuvastatin in AML therapy is warranted. 